\title{
Clustering South African Households Based on their Asset Status Using Latent Variable Models.
}

Damien McParland, Isobel Claire Gormley, Samuel J. Clark, Tyler H. McCormick, Chodziwadziwa Whiteson Kabudula and Mark A. Collinson.

\section{Derivation of the Full Conditional Posterior Distributions}

\section{Mixing Weights}

A Dirichlet prior distribution is used for the mixing weights $\underline{\pi}$. This is a conjugate prior which leads to a Dirichlet full conditional posterior.

$$
\begin{gathered}
h(\underline{\pi} \mid \ldots) \propto \prod_{g=1}^{G} \pi_{g}^{\left(n_{g}+\alpha_{g}\right)-1} \\
\Rightarrow \underline{\pi} \mid \ldots \sim \operatorname{Dirichlet}\left(n_{1}+\alpha_{1}, \ldots, n_{G}+\alpha_{G}\right)
\end{gathered}
$$

where $n_{g}=\sum_{i=1}^{N} \ell_{i g}$.

\section{Allocation Vectors}

A priori the allocation vectors, $\underline{\ell}_{i}$ are assumed to be $\operatorname{Multinomial}(1, \underline{\pi})$ distributed. Thus the full conditional posterior is also multinomial.

$$
\begin{aligned}
h\left(\underline{\ell}_{i} \mid \ldots\right) \propto \prod_{g=1}^{G}\left\{\pi_{g}\left[\prod_{j=1}^{O} \prod_{k=1}^{K_{j}} N\left(z_{i j} \mid \tilde{\hat{\lambda}}_{g j}^{T} \tilde{\tilde{\theta}}_{i}, 1\right)^{\mathbb{I}\left\{\gamma_{j, k-1}<z_{i j}<\gamma_{j, k}\right\}}\right]\right. \\
\left.\times\left[\prod_{j=O+1}^{J} \prod_{k=2}^{K_{j}-1} \prod_{s=1}^{3} N\left(z_{i j}^{k-1} \mid \underline{\tilde{\lambda}}_{g j}^{k-1^{T}} \underline{\tilde{\theta}}_{i}, 1\right)^{\mathbb{I}(\text { case } s)}\right]\right\}^{\ell_{i g}} \\
\Rightarrow \underline{\ell_{i}} \mid \ldots \sim \operatorname{Multinomial}(\underline{p})
\end{aligned}
$$


where

$$
\begin{aligned}
p_{g}= & \left\{\pi_{g}\left[\prod_{j=1}^{O} \prod_{k=1}^{K_{j}} N\left(z_{i j} \mid \tilde{\hat{\lambda}}_{g j}^{T} \tilde{\theta}_{i}, 1\right)^{\mathbb{I}\left\{\gamma_{j, k-1}<z_{i j}<\gamma_{j, k}\right\}}\right]\right. \\
& \left.\times\left[\prod_{j=O+1}^{J} \prod_{k=2}^{K_{j}-1} \prod_{s=1}^{3} N\left(z_{i j}^{k-1} \mid \underline{\tilde{\lambda}}_{g j}^{k-1^{T}} \underline{\hat{\theta}}_{i}, 1\right)^{\mathbb{I}(\text { case } s)}\right]\right\} \\
\times & \left\{\sum_{g=1}^{G} \pi_{g}\left[\prod_{j=1}^{O} \prod_{k=1}^{K_{j}} N\left(z_{i j} \mid \underline{\tilde{\lambda}}_{g j}^{T} \underline{\tilde{\theta}}_{i}, 1\right)^{\mathbb{I}\left\{\gamma_{j, k-1}<z_{i j}<\gamma_{j, k}\right\}}\right]\right. \\
& \left.\times\left[\prod_{j=O+1}^{J} \prod_{k=2}^{K_{j}-1} \prod_{s=1}^{3} N\left(z_{i j}^{k-1} \mid \underline{\tilde{\lambda}}_{g j}^{k-1^{T}} \underline{\tilde{\theta}}_{i}, 1\right)^{\mathbb{I}(\text { case } s)}\right]\right\}^{-1}
\end{aligned}
$$

\section{Threshold Parameters}

A uniform prior is specified for the threshold parameters, $\underline{\gamma}_{j}$, for ordinal items $j$. This leads to a uniform full conditional posterior distribution.

$$
\begin{aligned}
h\left(\gamma_{j, k} \mid \ldots\right) \propto & \prod_{i=1}^{N}\left[\mathbb{I}\left(y_{i j}=k\right) \mathbb{I}\left(\gamma_{j, k-1}<z_{i j}<\gamma_{j, k}\right)\right. \\
& \left.+\mathbb{I}\left(y_{i j}=k+1\right) \mathbb{I}\left(\gamma_{j, k}<z_{i j}<\gamma_{j, k+1}\right)\right]
\end{aligned}
$$

Note that $\underline{\gamma}_{j}$ is specified so that $\gamma_{j, 0}=-\infty, \gamma_{j, 1}=0$ and $\gamma_{j, K_{j}}=\infty$ for all ordinal items $j$. Also, $\underline{\gamma}_{j}$ is also constrained so that $\gamma_{j, 0}<\gamma_{j, 1}<\ldots<\gamma_{j, K_{j}}$.

The uniform full conditional distribution has support on the interval:

$$
\left(\max _{i}\left\{z_{i j} \mid y_{i j}=k\right\}, \min _{i}\left\{z_{i j} \mid y_{i j}=k+1\right\}\right)
$$

Samples from this distribution may be obtained using Gibbs sampling, however, to improve mixing Cowles (1996) proposes a Metropolis-Hastings approach which is adopted here.

Values $v_{j, k}$ are proposed for $\gamma_{j, k}\left(\right.$ for $\left.k=2, \ldots, K_{j}-1\right)$ from $\mathrm{N}^{T}\left(\gamma_{j, k}^{(t-1)}, \sigma_{M H}^{2}\right)$ truncated to the interval $\left(v_{j, k-1}, \gamma_{j, k+1}^{(t-1)}\right)$ where $\gamma_{j, k+1}^{(t-1)}$ is the value for $\gamma_{j, k+1}$ at iteration $(t-1)$. The threshold vector,$\underline{\gamma}_{j}$, is set equal to the proposed vector, $\underline{v}_{j}$, with probability $\beta=\min (1, R)$ where:

$$
\begin{aligned}
R= & \frac{\pi\left(\underline{v}_{j}\right)}{\pi\left(\underline{\gamma}_{j}^{(t-1)}\right)} \cdot \frac{q\left(\underline{\gamma}_{j}^{(t-1)} \mid \underline{v}_{j}\right)}{q\left(\underline{v}_{j} \mid \underline{\gamma}_{j}^{(t-1)}\right)} \\
= & \prod_{i=1}^{N} \prod_{g=1}^{G}\left[\frac{\Phi\left(v_{j, y_{i j}}-\underline{\tilde{\lambda}}_{g j}^{T} \underline{\tilde{\theta}}_{i}\right)-\Phi\left(v_{j, y_{i j}-1}-\underline{\tilde{\lambda}}_{g j}^{T} \underline{\tilde{\theta}}_{i}\right)}{\Phi\left(\gamma_{j, y_{i j}}^{(t-1)}-\tilde{\tilde{\lambda}}_{g j}^{T} \tilde{\tilde{\theta}}_{i}\right)-\Phi\left(\gamma_{j, y_{i j}-1}^{(t-1)}-\underline{\tilde{\lambda}}_{j j}^{T} \tilde{\tilde{\theta}}_{i}\right)}\right]^{\ell_{i g}} \\
& \times \prod_{k=2}^{K_{j}-1} \frac{\Phi\left[\left(\gamma_{j, k+1}^{(t-1)}-\gamma_{j, k}^{(t-1)}\right) / \sigma_{M H}\right]-\Phi\left[\left(v_{j, k-1}-\gamma_{j, k}^{(t-1)}\right) / \sigma_{M H}\right]}{\Phi\left[\left(v_{j, k+1}-v_{j, k}\right) / \sigma_{M H}\right]-\Phi\left[\left(\gamma_{j, k-1}^{(t-1)}-v_{j, k}\right) / \sigma_{M H}\right]}
\end{aligned}
$$


The tuning parameter $\sigma_{M H}^{2}$ is selected to achieve appropriate acceptance rates.

\section{Latent Traits}

A priori the latent traits, $\underline{\theta}_{i}$, are assumed to be standard multivariate Gaussian distributed. The resulting full conditional posterior is also multivariate Gaussian.

$$
\begin{aligned}
& h\left(\underline{\theta}_{i} \mid \ldots\right) \propto \prod_{d=1}^{D} \mathrm{~N}\left(z_{i d} \mid \tilde{\lambda}_{g d}^{T} \tilde{\theta}_{i}, 1\right) \operatorname{MVN}_{q}\left(\underline{0}, \mathbf{I}_{q}\right) \\
& \propto \exp \left\{-\frac{1}{2} \sum_{d=1}^{D}\left(z_{i d}-\underline{\tilde{\lambda}}_{g d}^{T} \underline{\theta}_{i}\right)^{2}\right\} \exp \left\{-\frac{1}{2} \underline{\theta}_{i}^{T} \underline{\theta}_{i}\right\} \\
& \propto \exp \left\{-\frac{1}{2} \underline{\theta}_{i}^{T}\left[\Lambda_{g}^{T} \Lambda_{g}+\mathbf{I}_{q}\right] \underline{\theta}_{i}+\left[\left(\underline{z}_{i}-\underline{\mu}_{g}\right)^{T} \Lambda_{g}\right] \underline{\theta}_{i}\right\} \\
& \Rightarrow \underline{\theta}_{i} \mid \ldots \sim \operatorname{MVN}_{q}\left\{\left[\Lambda_{g}^{T} \Lambda_{g}+\mathbf{I}_{q}\right]^{-1}\left[\Lambda_{g}^{T}\left(\underline{z}_{i}-\underline{\mu}_{g}\right)\right],\left[\Lambda_{g}^{T} \Lambda_{g}+\mathbf{I}_{q}\right]^{-1}\right\}
\end{aligned}
$$

\section{Loadings Matrix and Mean}

A multivariate Gaussian prior is specified for $\tilde{\lambda}_{g d}$. This is a conjugate prior and so leads to a multivariate Gaussian full conditional posterior.

$$
\begin{aligned}
& h\left(\underline{\tilde{\lambda}}_{g d} \mid \ldots\right) \propto \prod_{i=1}^{N}\left[\mathrm{~N}\left(z_{i d} \mid \underline{\tilde{\lambda}}_{g d}^{T} \underline{\tilde{\theta}}_{i}, 1\right)\right]^{\ell_{i g}}\left[\operatorname{MVN}_{(q+1)}\left(\underline{\tilde{\lambda}}_{g d} \underline{\mu}_{\lambda}, \Sigma_{\lambda}\right)\right] \\
& \propto \exp \left\{-\frac{1}{2} \sum_{i: \ell_{i g}=1}\left(z_{i d}-\underline{\tilde{\lambda}}_{g d}^{T} \underline{\tilde{\theta}}_{i}\right)^{2}\right\} \exp \left\{-\frac{1}{2}\left(\underline{\tilde{\lambda}}_{g d}-\underline{\mu}_{\lambda}\right)^{T} \Sigma_{\lambda}^{-1}\left(\underline{\tilde{\lambda}}_{g d}-\underline{\mu}_{\lambda}\right)\right\} \\
& \propto \exp \left\{-\frac{1}{2} \tilde{\hat{\lambda}}_{g d}^{T}\left[\Sigma_{\lambda}^{-1}+\tilde{\Theta}_{g}^{T} \tilde{\Theta}_{g}\right] \underline{\tilde{\lambda}}_{g d}+\underline{\tilde{\lambda}}_{g j}^{T}\left[\tilde{\Theta}_{g}^{T} \underline{z}_{g d}+\Sigma_{\lambda}^{-1} \underline{\mu}_{\lambda}\right]\right\} \\
& \Rightarrow \quad \underline{\hat{\lambda}}_{g d} \mid \ldots \sim \operatorname{MVN}_{(q+1)}\left\{\left[\Sigma_{\lambda}^{-1}+\tilde{\Theta}_{g}^{T} \tilde{\Theta}_{g}\right]^{-1}\left[\tilde{\Theta}_{g}^{T} \underline{z}_{g d}+\Sigma_{\lambda}^{-1} \underline{\mu}_{\lambda}\right],\left[\Sigma_{\lambda}^{-1}+\tilde{\Theta}_{g}^{T} \tilde{\Theta}_{g}\right]^{-1}\right\}
\end{aligned}
$$

where

$$
\underline{z}_{g d}=\left\{z_{i d}\right\} \text { for all individuals in group } g \text {. }
$$

\section{References}

Cowles, M. K. (1996). Accelerating monte carlo markov chain convergence for cumulative-link generalized linear models. Statistics and Computing 6(2), 101111. 